14-21 days, while 18 (51\%) received <14 days and 8 (23\%) received more than 21 days. Among 735 others, 701 (95\%) had symptoms compatible with HSV infection; these included vesicular lesions in $10(1 \%)$ and seizure in 244 (33\%). Overall, 172 (23\%) were ever tested for HSV, including 7/10 (70\%) with vesicular lesions and 69/ $244(28 \%)$ with seizure. At least $98(13 \%)$ received some acyclovir, including $2 / 10(20 \%)$ with vesicular lesions and 18/244 (7\%) with seizure. Of these, 32 (4\%) infants received acyclovir for $\geq 7$ days.

Conclusions Our results reveal variations in clinical care of infants with possible neonatal HSV infection. Among cases, duration of antiviral therapy rarely followed treatment recommendations. Among symptomatic infants, most with vesicular lesions but few with seizures were ever tested for HSV. These variations suggest opportunities for clinical quality improvements.

\section{P3-S2.03 CLINICAL EVALUATION OF THE BD HSV1 OX ASSAY FOR THE DIRECT QUALITATIVE TESTING OF HSV1 AS COMPARED TO VIRAL CULTURE AND A LABORATORY- BASED PCR ASSAY USING MALE AND FEMALE EXTERNAL ANOGENITAL LESIONS}

doi:10.1136/sextrans-2011-050108.447

\begin{abstract}
${ }^{1} \mathrm{~A}$ Pantone, ${ }^{1} \mathrm{~B}$ Van Der Pol, ${ }^{1} \mathrm{~J}$ Williams, ${ }^{2} \mathrm{~L}$ Corey, ${ }^{3} \mathrm{E}$ Hook, ${ }^{4} \mathrm{~B}$ Body, ${ }^{5} \mathrm{~S}$ Taylor, ${ }^{6} \mathrm{P}$ Fine, ${ }^{7} \mathrm{~S}$ Ginde, ${ }^{8} \mathrm{~J}$ Lebed. ${ }^{1}$ Indiana University School of Medicine, Indianapolis, USA; ${ }^{2}$ University of Washington, Seattle, USA; ${ }^{3}$ University of Alabama Birmingham, Birmingham, USA; ${ }^{4}$ LabCorp, Burlington, USA; ${ }^{5}$ LSU Health Science Center, New Orleans, USA; ${ }^{6}$ Planned Parenthood Gulf Coast, Houston, USA; ${ }^{7}$ Planned Parenthood of the Rocky Mountains, Denver, USA; ${ }^{8}$ Planned Parenthood Southeastern Pennsylvania, Philadelphia, USA
\end{abstract}

Background To compare the performance characteristics of the $\mathrm{BD}$ ProbeTecTM HSV1 Qx Assay* on the BD ViperTM System in Extracted Mode to viral culture and a well-characterised molecular assay for the detection of HSV1. External anogenital lesions were sampled with two different collection devices: a universal viral transport (UVT) kit and a BD Ox swab (OS) kit*.

Methods Eleven geographically diverse clinical centers participated in the study, with nine of the sites enrolling participants. The UVT was collected first followed by the OS specimen. A portion of each UVT specimen was transferred to a Qx Diluent tube (diluted UVT) and a cryovial. The remainder of the UVT in the original tube was frozen at $-70^{\circ} \mathrm{C}$ and sent to one of two sites for HSV viral culture using the ELVIS ${ }^{\circledR} H S V$ ID and D3 Typing Test System (Diagnostic Hybrids, Inc). The diluted UVT and OS specimens were shipped to one of three sites for HSV testing on the BD Viper. The UVT aliquot in the cryovial was stored at $-70^{\circ} \mathrm{C}$ and shipped to the University of Washington for PCR testing for HSV.

Results Subjects $(n=508)$ were enrolled from February to August of 2010 with 312 UVT and 308 OS samples available for comparison to ELVIS culture, and 506 and 502 samples, respectively, for comparison to the PCR assay. Samples positive for HSV2 by viral culture did not have results for HSV1 per the ELVIS package insert and were excluded from further analysis. The sensitivity and specificity of the BD HSV1 Qx Assay as compared to ELVIS culture and the positive (PPA) and negative per cent agreement (NPA) of the assay compared to the HSV PCR assays were determined for both specimen types see Abstract P3-S2.03 table 1.

Conclusions The BD HSV1 Qx Assay on the BD Viper System had excellent agreement with viral culture and the lab developed PCR assay, which is currently recognised as one of the best available tests for the detection of HSV1. A commercially available molecular-based assay for the detection of HSV would not only improve detection and reduce turn-around time on results, but may also obviate the need for stringent transport conditions required for HSV culture. *Product not for sale, for investigational use only in the US.
Abstract P3-S2.03 Table 1 HSV1

\begin{tabular}{|c|c|c|c|c|}
\hline \multirow[b]{3}{*}{ Specimen type } & \multicolumn{4}{|c|}{ HSV1 $0^{x}$ assay performance } \\
\hline & \multicolumn{2}{|c|}{ Compared to ELVIS HSV1 culture } & \multicolumn{2}{|c|}{ Compared to HSV1 PCR assay } \\
\hline & Sensitivity & Specificity & PPA & NPA \\
\hline Diluted UVT & $96.8 \%(60 / 62)$ & $97.6 \%(244 / 250)$ & $98.6 \%(71 / 72)$ & $100 \%(434 / 434)$ \\
\hline $\mathrm{Q}^{\mathrm{x}}$ Swab (QS) & $96.7 \%(59 / 61)$ & $95.1 \%(235 / 247)$ & $100 \%(71 / 71)$ & $98.8 \%(426 / 431)$ \\
\hline
\end{tabular}

P3-S2.04 CLINICAL EVALUATION OF THE BD HSV2 OX ASSAY FOR THE DIRECT QUALITATIVE TESTING OF HSV2 AS COMPARED TO VIRAL CULTURE AND A LABORATORYBASED PCR ASSAY USING MALE AND FEMALE EXTERNAL ANOGENITAL LESIONS

doi:10.1136/sextrans-2011-050108.448

${ }^{1} \mathrm{~L}$ Hires, ${ }^{1} \mathrm{~B}$ Van Der Pol, ${ }^{2} \mathrm{~J}$ Williams, ${ }^{3} \mathrm{~L}$ Corey, ${ }^{4} \mathrm{E}$ W Hook, ${ }^{5} \mathrm{M}$ Nye, ${ }^{6} \mathrm{~S}$ Taylor, ${ }^{7} \mathrm{M}$ Martens, ${ }^{8}$ L Mena, ${ }^{9}$ T Warren. 'Indiana University, School of Medicine, Indianapolis, USA; ${ }^{2}$ Indiana University, Indianapolis, USA; ${ }^{3}$ University of Washington, Seattle, USA; ${ }^{4}$ University of Alabama, Birmingham, USA; ${ }^{5}$ LabCorp, Burlington, USA; ${ }^{6}$ LSU Health Science Center, New Orleans, USA; 7 Planned Parenthood of Arkansas and Eastern Oklahoma, Tulsa, USA; ${ }^{8}$ University of Mississippi, Medical Center, Jackson, USA; ${ }^{9}$ Westover Heights, Portland, USA

Background To compare the performance characteristics of the BD ProbeTecTM HSV2 Ox Assay* on the BD ViperTM System in Extracted Mode to viral culture and a well-characterised molecular assay for the detection of HSV2. External anogenital lesions were sampled with two different collection devices: a universal viral transport (UVT) kit and a BD Ox swab (OS) kit*

Methods Eleven geographically diverse clinical centers participated in the study, with nine of the sites enrolling participants. The UVT was collected first followed by the OS specimen. A portion of each UVT specimen was transferred to a Ox Diluent Tube (diluted UVT) and a cryovial. The remainder of the UVT in the original tube was frozen at $-70^{\circ} \mathrm{C}$ and sent to one of two sites for HSV viral culture using the ELVIS®HSV ID and D3 Typing Test System (Diagnostic Hybrids, Inc). The diluted UVT and OS specimens were shipped to one of three sites for HSV testing on the BD Viper. The UVT aliquot in the cryovial was stored at $-70^{\circ} \mathrm{C}$ and shipped to the University of Washington for PCR testing for HSV.

Results Subjects $(n=508)$ were enrolled between February and August of 2010 with 501 UVT and 498 OS samples available for comparison to ELVIS culture, and 506 and 503 samples, respectively, for comparison to the PCR assay. The sensitivity and specificity of the BD HSV2 Ox Assay were compared to ELVIS culture and the positive (PPA) and negative (NPA) per cent agreement of the assay was compared to the HSV PCR assays for both specimen types. Of the BD HSV2 Ox Assay positive, culture negative samples, 46/51 (90.2\%) of the UVT and 49/60 (81.7\%) of the QS were positive by HSV2 PCR see Abstract P3-S2.04 table 1.

Conclusions The BD HSV2 Ox Assay on the BD Viper had excellent agreement with the lab developed PCR assay for diagnosis of HSV2, which is currently recognised as one of the best available tests for the detection of HSV. A commercially available molecular-based assay for the detection of HSV would not only improve detection and reduce turn-around time on results, but may also obviate the need for stringent transport conditions required for HSV culture. *Product not for sale, for investigational use only in the USA.

Abstract P3-S2.04 Table 1 HSV2

\begin{tabular}{|c|c|c|c|c|}
\hline \multirow[b]{3}{*}{ Specimen type } & \multicolumn{4}{|c|}{ HSV2 $0^{x}$ assay performance } \\
\hline & \multicolumn{2}{|c|}{ Compared to ELVIS HSV2 culture } & \multicolumn{2}{|c|}{ Compared to HSV2 PCR assay } \\
\hline & Sensitivity & Specificity & PPA & NPA \\
\hline Diluted UVT & $98.4 \%(186 / 189)$ & $83.7 \%(261 / 312)$ & $97.5 \%(237 / 243)$ & $98.1 \%(258 / 263)$ \\
\hline $0^{x}$ Swab (0S) & $98.4 \%(186 / 189)$ & $80.6 \%(249 / 309)$ & $98.8 \%(240 / 243)$ & $95.8 \%(249 / 260)$ \\
\hline
\end{tabular}

